\title{
microRNA expression profiles of scar and normal tissue from patients with posterior urethral stricture caused by pelvic fracture urethral distraction defects
}

\author{
KAILE ZHANG ${ }^{1,2^{*}}$, JUN CHEN $^{1 *}$, DONGLIANG ZHANG ${ }^{1 *}$, LIN WANG $^{1}$, WEIXIN ZHAO ${ }^{2}$, DAVID YUN-TE LIN ${ }^{3}$, \\ RONG CHEN $^{1}$, HONG XIE ${ }^{1}$, XIAOYONG HU ${ }^{1}$, XIAOLAN FANG ${ }^{3}$ and QIANG FU ${ }^{1}$ \\ ${ }^{1}$ Department of Urology, Affiliated Sixth People's Hospital, Shanghai JiaoTong University, Shanghai 200233, P.R. China; \\ ${ }^{2}$ Wake Forest Institute for Regenerative Medicine, Winston-Salem, NC 27157; \\ ${ }^{3}$ New York Genome Center, New York, NY 10013, USA
}

Received October 20, 2017; Accepted January 23, 2018

DOI: $10.3892 /$ ijmm.2018.3487

\begin{abstract}
Pelvic fracture urethral distraction defect (PFUDD) seriously affects the quality of life of patients. At present, there are few effective drug treatments available for PFUDD-induced urethral stricture, which is associated with fibrosis and scar formation in urethra lumen. Emerging evidence suggests that microRNAs (miRNAs/miRs) may be involved in the regulation of fibrosis, and analysis of miRNA expression profiles in urethral scar and normal urethra tissues may therefore benefit the discovery of novel treatments for urethral stricture with micro invasive procedures. In the present study, miRNA sequencing and quantitative polymerase chain reaction (qPCR) validation using paired scar and normal tissues from patients with PFUDD, and functional analysis of the miRNAs involved in the fibrosis associated signaling pathway was performed. A total of 94 differentially expressed miRNAs were identified in the scar tissue of patients with PFUDD. Among them, 26 miRNAs had significantly altered expression in the scar tissue compared with the normal tissue from the same patient. qPCR validation confirmed that miR-129-5p was overexpressed
\end{abstract}

Correspondence to: Dr Qiang Fu, Department of Urology, Affiliated Sixth People's Hospital, Shanghai JiaoTong University, 600 Yishan Road, Xuhui, Shanghai 200233, P.R. China

E-mail: jamesqfu@aliyun.com

Dr Xiaolan Fang, New York Genome Center, 101 Avenue of Americas, New York, NY 10013, USA

E-mail: afang@nygenome.org

*Contributed equally

Abbreviations: PFUDD, pelvic fracture urethral distraction defects; ECM, extracellular matrix; DVIU, direct visual internal urethrotomy; EMT, epithelial-to-mesenchymal transition

Key words: Urethral stricture, microRNA sequencing and profiling, molecular mechanism, precision medicine in scar tissue. The TGF- $\beta$ pathway-associated functions of a total of 5 miRNAs (hsa-miR-129-5p, hsa-miR-135a-5p, hsa-miR-363-3p, hsa-miR-6720-3p and hsa-miR-9-5p) were further analyzed, as well as their key molecular targets and functional mechanisms in signaling regulation. To conclude the miRNA sequencing indicated a significantly altered expression of hsa-miR-129-5p, hsa-miR-135a-5p, hsa-miR-363-3p, hsa-miR-6720-3p and hsa-miR-9-5p in patients with PFUDD. These miRNAs and their potential target genes were associated with fibrosis in several diseases, and the data from the present study may help explore potential miRNA targets for future precision treatments for urethral stricture.

\section{Introduction}

Pelvic fracture urethral distraction defect (PFUDD) seriously affects voiding function and the quality of life of patients (1). Urethral defects are healed by fibroblasts, but overexpression of extracellular matrix (ECM) components in the resultant scar tissue may cause a reduction or complete blockage in the urethral caliber and impairment of the flow of urine (2). Open urethroplasty of urethral strictures is performed worldwide and has become the gold standard for the treatment of urethral strictures. However, this surgery is often performed in high-volume urethral referral centers, and only surgeons with multiple years of training are able to obtain high $(\sim 95 \%)$ success rates (3). Overall, the success rate of urethral reconstructive surgeries remains relatively low globally (short-term $79-88 \%$, long term 42-86\%) (4,5). Reconstructive surgery may also result in serious morbidity at the defect location and the donor site, including infection, nerve injury and difficulties in opening the mouth $(2,6)$. To minimize side-effects and complications, micro invasive surgeries [for example, direct visual internal urethrotomy (DVIU)] has been used to treat posterior urethral stricture caused by PFUDD. DVIU is commonly performed in patients with urethral stricture and remains the first choice in secondary centers with limited equipment or those that lack experienced surgeons. However, high recurrence and poor long-term curative rates have been associated with this technique, and its effectiveness remains questionable 
Table I. Baseline patient characteristics.

\begin{tabular}{llll}
\hline Patient number & Age & Sex & Health status \\
\hline 1 & 59 & Male & PFUDD \\
2 & 50 & Male & PFUDD \\
3 & 16 & Male & PFUDD \\
4 & 43 & Male & PFUDD \\
5 & 44 & Male & PFUDD
\end{tabular}

PFUDD, pelvic fracture urethral distraction defects.

among urologists $(7,8)$. Thus, there is an urgent need for novel techniques and complementary strategies to prevent fibrosis and scar formation in patients following surgery. Although the local injection and dilution of steroids and other anti-fibrosis reagents were introduced as an adjuvant treatment for DVIU, a previous systematic review and meta-analysis by our group has demonstrated that these drugs are not efficient or effective enough to decrease the recurrence rate (7).

Gene therapy has emerged as a promising treatment option for a number of diseases. Accumulating evidence indicates that microRNAs (miRNAs/miRs) are involved in multiple disease processes, including the pathogenesis of fibrosis in the skin and liver (9). Our goal is to add to the information regarding miRNA dysregulation in urethral fibrosis to broaden our understanding of the underlying pathological mechanisms and discover novel treatment targets. By conducting miRNA sequencing, temporal miRNA expression profiles were generated in urethral scar and normal urethral tissues, and potential gene targets and pathways involved in fibrosis were identified in patients suffering from PFUDD. This established miRNA profile may provide further insight into the involvement of miRNAs and their functions in the development and progression of pathological conditions.

\section{Materials and methods}

Urethral scar samples. All procedures performed in studies involving human participants were in accordance with the ethical standards of the institutional research committee and in accordance with the 1964 Declaration of Helsinki and its later amendments or comparable ethical standards. The present study was approved by the Ethics Committee of Shanghai Sixth People's Hospital (Shanghai, China). Informed consent was obtained from all patients. Scar and normal urethral tissues were collected from patients with urethral stricture undergoing urethroplasty $(n=5)$. All subjects were male with an age range of 16-59 years. Baseline patient information is summarized in Table I.

A total of 3 pairs of samples were used for miRNA sequencing, and 3 pairs were used for quantitative polymerase chain reaction (qPCR) verification (one patient's tissue was used in both miRNA sequencing and qPCR). The etiology of patients with urethral stricture was PFUDD. All the participating patients underwent primary surgery. The mean length of the stricture was $1.5 \mathrm{~cm}$ and the locations were all at the membranous segment of the urethra. Samples were harvested following surgery, sectioned and stored at $-80^{\circ} \mathrm{C}$ until the process of RNA extraction.

RNA extraction and miRNA sequencing analysis. Total RNA from each sample (Table II) was used to prepare the miRNA sequencing library, which included the following steps: i) 3'-adaptor ligation; ii) 5'-adaptor ligation; iii) cDNA synthesis; iv) PCR amplification; v) size selection of $\sim 135-155 \mathrm{bp}$ PCR amplified fragments (corresponding to $15-35$ nt small RNAs).

The TruSeq SR Cluster kit (cat. no. GD-402-4001; Illumina, Inc., San Diego, CA, USA) was used for cluster generation in an Illumina cBOT instrument, following the manufacturer's protocol. Sequencing was performed on an Illumina HiSeq ${ }^{\circledR} 2000$ instrument (Illumina, Inc.) using a TruSeq Rapid SBS kit (cat. no. FC-402-4002; Illumina, Inc.).

Following sequencing, the Solexa CHASTITY quality filtered reads were harvested as clean reads. Adaptor sequences were trimmed, and the retained reads ( $\geq 15 \mathrm{nt}$ ) were aligned to merged pre-miRNA databases (known pre-miRNAs from miRBase v21 (10-14) plus the newly predicted pre-miRNAs) using Novoalign software v2.07.11 [(Novocraft Technologies Sdn Bhd, Selangor, Malaysia) maximum mismatch was 1, and low reads (total reads $<2$ ) were discarded]. To characterize the isomiR variability, sequences matching each miRNA precursor(s) in the mature miRNA region $\pm 4 \mathrm{nt}$ ( $\leq 1$ mismatch) were accepted as mature miRNA isomiRs and grouped according to the 5-prime (5p) or 3-prime (3p) arm of the precursor hairpin. The number of mapped tags was defined as the raw expression level of the specific miRNAs. Transcripts per million values were generated based on the total number of aligned tags for normalization. The

Table II. RNA quality control characteristics for microRNA sequencing samples.

\begin{tabular}{lcccrr}
\hline Sample ID & OD260/280 ratio & OD260/230 ratio & Conc. (ng/ $\mu$ l) & Volume $(\mu 1)$ & Quantity (ng) \\
\hline Normal 1 & 1.87 & 2.40 & 316.68 & 30 & 9500.40 \\
Scar 1 & 1.85 & 2.38 & 282.52 & 40 & 11300.80 \\
Normal 2 & 1.86 & 2.41 & 182.11 & 20 & 3642.20 \\
Scar 2 & 1.88 & 2.39 & 237.14 & 40 & Pass \\
Normal 3 & 1.81 & 2.40 & 144.77 & 10 & 9485.60 \\
Scar 3 & 1.88 & 2.30 & 267.73 & 40 & 1447.70 \\
\hline
\end{tabular}

an Sample ID, 1, 2 and 3 indicate specific patients (for example, 1 for Patient 1). OD, optical density; QC, quality control. 
Table III. MicroRNA primers for quantitative real-time PCR.

Primer name

Primer sequence $\left(5^{\prime}-3^{\prime}\right)$

\section{U6-S}

U6-A

general control primer-A hsa-miR-129-5p-RT

hsa-miR-129-5p-S

hsa-miR-9-5p-RT

hsa-miR-9-5p-S

hsa-miR-9-3p-RT

hsa-miR-9-3p-S

hsa-miR-183-5p-RT

hsa-miR-183-5p-S

hsa-miR-6720-3p-RT

hsa-miR-6720-3p-S

hsa-miR-96-5p-RT

hsa-miR-96-5p-S

hsa-miR-486-5p-RT

hsa-miR-486-5p-S

hsa-miR-363-3p-RT

hsa-miR-363-3p-S

hsa-miR-135a-5p-RT

hsa-miR-135a-5p-S

hsa-miR-374a-3p-RT

hsa-miR-374a-3p-S

hsa-miR-374b-3p-RT

hsa-miR-374b-3p-S

hsa-miR-941-RT

hsa-miR-941-S

hsa-miR-3158-3p-RT

hsa-miR-3158-3p-S

hsa-miR-29c-3p-RT

hsa-miR-29c-3p-S

hsa-miR-10a-3p-RT

hsa-miR-10a-3p-S

hsa-miR-182-5p-RT

hsa-miR-182-5p-S

hsa-miR-106a-5p-RT

hsa-miR-106a-5p-S

hsa-miR-4284-RT

hsa-miR-4284-S

has-mir192-5p-RT

has-mir192-5p-S

hsa-miR-26a-5p-RT

hsa-miR-26a-5p-S

hsa-miR-223-3p-RT

hsa-miR-223-3p-S

hsa-miR-92a-3p-RT

hsa-miR-92a-3p-S

hsa-miR-29b-3p-RT

hsa-miR-29b-3p-S

hsa-miR-125b-5p

hsa-miR-125b-5p

hsa-miR-194-5p-RT
CTCGCTTCGGCAGCACA

AACGCTTCACGAATTTGCGT

TGGTGTCGTGGAGTCG

CTCAACTGGTGTCGTGGAGTCGGCAATTCAGTTGAGGCAAGCCC

ACACTCCAGCTGGGCTTTTTGCGGTCTGG

CTCAACTGGTGTCGTGGAGTCGGCAATTCAGTTGAGTCATACAG

ACACTCCAGCTGGGTCTTTGGTTATCTAGCT

CTCAACTGGTGTCGTGGAGTCGGCAATTCAGTTGAGACTTTCGG

ACACTCCAGCTGGGATAAAGCTAGATAACC

CTCAACTGGTGTCGTGGAGTCGGCAATTCAGTTGAGAGTGAATT

ACACTCCAGCTGGGTATGGCACTGGTAGAA

CTCAACTGGTGTCGTGGAGTCGGCAATTCAGTTGAGTCTACCAG

ACACTCCAGCTGGGCGCGCCTGCAGGAACT

CTCAACTGGTGTCGTGGAGTCGGCAATTCAGTTGAGAGCAAAAA

ACACTCCAGCTGGGTTTGGCACTAGCACATT

CTCAACTGGTGTCGTGGAGTCGGCAATTCAGTTGAGCTCGGGGC

ACACTCCAGCTGGGTCCTGTACTGAGCTGC

CTCAACTGGTGTCGTGGAGTCGGCAATTCAGTTGAGTACAGATG

ACACTCCAGCTGGGAATTGCACGGTATCCA

CTCAACTGGTGTCGTGGAGTCGGCAATTCAGTTGAGTCACATAG

ACACTCCAGCTGGGTATGGCTTTTTATTCCT

CTCAACTGGTGTCGTGGAGTCGGCAATTCAGTTGAGAATTACAA

ACACTCCAGCTGGGCTTATCAGATTGTATT

CTCAACTGGTGTCGTGGAGTCGGCAATTCAGTTGAGAATGATAA

ACACTCCAGCTGGGCTTAGCAGGTTGTATT

CTCAACTGGTGTCGTGGAGTCGGCAATTCAGTTGAGGCACATGT

ACACTCCAGCTGGGCACCCGGCTGTGTGCAC

CTCAACTGGTGTCGTGGAGTCGGCAATTCAGTTGAGGTCCTGCA

ACACTCCAGCTGGGAAGGGCTTCCTCTCTG

CTCAACTGGTGTCGTGGAGTCGGCAATTCAGTTGAGTAACCGAT

ACACTCCAGCTGGGTAGCACCATTTGAAAT

CTCAACTGGTGTCGTGGAGTCGGCAATTCAGTTGAGTATTCCCC

ACACTCCAGCTGGGCAAATTCGTATCTAGG

CTCAACTGGTGTCGTGGAGTCGGCAATTCAGTTGAGAGTGTGAG

ACACTCCAGCTGGGTTTGGCAATGGTAGAACT

CTCAACTGGTGTCGTGGAGTCGGCAATTCAGTTGAGCTACCTGC

ACACTCCAGCTGGGAAAAGTGCTTACAGTGC

CTCAACTGGTGTCGTGGAGTCGGCAATTCAGTTGAGATGGGGTG

ACACTCCAGCTGGGGGGCTCACATCA

CTCAACTGGTGTCGTGGAGTCGGCAATTCAGTTGAGGGCTGTCA

ACACTCCAGCTGGGCTGACCTATGAATTG

CTCAACTGGTGTCGTGGAGTCGGCAATTCAGTTGAGAGCCTATC

ACACTCCAGCTGGGTTCAAGTAATCCAGGA

CTCAACTGGTGTCGTGGAGTCGGCAATTCAGTTGAGTGGGGTAT

ACACTCCAGCTGGGTGTCAGTTTGTCAAAT

CTCAACTGGTGTCGTGGAGTCGGCAATTCAGTTGAGACAGGCCG

ACACTCCAGCTGGGTATTGCACTTGTCCCG

CTCAACTGGTGTCGTGGAGTCGGCAATTCAGTTGAGAACACTGA

ACACTCCAGCTGGGTAGCACCATTTGAAATC

CTCAACTGGTGTCGTGGAGTCGGCAATTCAGTTGAGTCACAAGT

ACACTCCAGCTGGGTCCCTGAGACCCTAAC

CTCAACTGGTGTCGTGGAGTCGGCAATTCAGTTGAGTCCACATG 
Table III. Continued.

Primer name

Primer sequence (5'-3')

hsa-miR-194-5p-S
hsa-miR-20a-5p-RT
hsa-miR-20a-5p-S
hsa-miR-128-3p-RT
hsa-miR-128-3p-S
hsa-miR-654-5p-S
hsa-miR-654-5p-RT
hsa-miR-31-3p-S
hsa-miR-31-3p-RT
hsa-miR-329-3p-S
hsa-miR-329-3p-RT
hsa-let-7b-3p-S
hsa-let-7b-3p-RT
hsa-miR-433-3p-S
hsa-miR-433-3p-RT
hsa-miR-379-3p-S
hsa-miR-379-3p-RT
hsa-miR-744-5p-S
hsa-miR-744-5p-RT
hsa-miR-29c-5p-S
hsa-miR-29c-5p-RT

\author{
ACACTCCAGCTGGGTGTAACAGCAACTCCA \\ CTCAACTGGTGTCGTGGAGTCGGCAATTCAGTTGAGCTACCTGC \\ ACACTCCAGCTGGGTAAAGTGCTTATAGTGC \\ CTCAACTGGTGTCGTGGAGTCGGCAATTCAGTTGAGAAAGAGAC \\ ACACTCCAGCTGGGTCACAGTGAACCGGT \\ ACACTCCAGCTGGGTGGTGGGCCGCAGAAC \\ CTCAACTGGTGTCGTGGAGTCGGCAATTCAGTTGAGGCACATGT \\ ACACTCCAGCTGGGTGCTATGCCAACATAT \\ CTCAACTGGTGTCGTGGAGTCGGCAATTCAGTTGAGATGGCAAT \\ ACACTCCAGCTGGGAACACACCTGGTTAAC \\ CTCAACTGGTGTCGTGGAGTCGGCAATTCAGTTGAGAAAGAGGT \\ ACACTCCAGCTGGGCTATACAACCTACTGC \\ CTCAACTGGTGTCGTGGAGTCGGCAATTCAGTTGAGAGGAAGGC \\ ACACTCCAGCTGGGATCATGATGGGCTCCT \\ CTCAACTGGTGTCGTGGAGTCGGCAATTCAGTTGAGACACCGAG \\ ACACTCCAGCTGGGTATGTAACATGGTCCA \\ CTCAACTGGTGTCGTGGAGTCGGCAATTCAGTTGAGAGTTAGTG \\ ACACTCCAGCTGGGTGCGGGGCTAGGGCTA \\ CTCAACTGGTGTCGTGGAGTCGGCAATTCAGTTGAGTGCTGTTA \\ ACACTCCAGCTGGGTGACCGATTTCTCCTG \\ CTCAACTGGTGTCGTGGAGTCGGCAATTCAGTTGAGGAACACCA
}

most abundant isomiR, mature miRNA (annotated in miRBase) and all isoforms of miRNA ( $5 p$ or $3 p$ ) were used for miRNA expression quantification. For comparison of miRNA expression profiles between two groups (scar tissue vs. normal tissue), fold change and P-value were calculated to identify miRNAs with differentiated expression.

Reverse transcription (RT)-qPCR for the validation of $m i R N A s$. A total of 3 pairs of urethral samples were used for qPCR validation of miRNA expression. The primers used for PCR were designed with Primer Premier software (version 5.0; Premier Biosoft International, Palo Alto, CA, USA) (primer sequence details are summarized in Table III). cDNA synthesis was performed on a GeneAmp PCR System 9700 (Applied Biosystems; Thermo Fisher Scientific, Inc., Waltham, MA, USA) using a RevertAid First Strand cDNA Synthesis kit, cat. no. K1622 (Thermo Fisher Scientific, Inc.) according to the manufacturer's protocols. qPCR was performed on a ViiA 7 Real-time PCR System (Applied Biosystems; Thermo Fisher Scientific, Inc.) using a PowerUp SYBR Green Master Mix, (cat. no. A25778, Thermo Fisher Scientific, Inc.). qPCR was conducted at: i) $95^{\circ} \mathrm{C}$ for $10 \mathrm{~min}$, ii) $95^{\circ} \mathrm{C}$ for $15 \mathrm{sec}$ and iii) $60 \mathrm{sec}$ at $>60^{\circ} \mathrm{C}$ for 40 cycles. The fold change for each miRNA was calculated using the $2^{-\Delta \Delta C q}$ method (15).

Bioinformatics analysis. Hierarchical clustering was performed. miRNAtargetprediction wasperformedby TargetScan,miRanda, and microcosm with DAVID Bioinformatics Resources 6.7 (https://david-d.ncifcrf.gov/home) (16-22). Enrichment analysis for Gene Ontology (GO) and Kyoto Encylopedia of Genes and Genomes (KEGG) pathways was performed using the topGO package in R (https://www.bioconductor .org/packages/3.7/bioc/html/topGO.html) (20-22).

Statistical analysis. Comparisons between average fold changes of different miRNAs were performed using Student's t-tests (using the T.TEST function in Microsoft Excel; Microsoft Corporation, Redmond, WA, USA). For analysis based on miRNA sequencing only, the t-tests used a two-tailed distribution and the comparison was paired for expression in normal and scar tissues ( $n=3$; Table IV). For analysis based on both miRNA sequencing and qPCR validation (Table V), the t-tests used a two-tailed distribution and the comparison was paired for expression evaluated by the two methods [miRNA sequencing and $\mathrm{qPCR}, \mathrm{n}=7$ (for upregulated miRNAs) and $\mathrm{n}=15$ (for downregulated miRNAs)].

Histological evaluation. Freshly harvested scar and normal tissue were fixed in $4 \%$ paraformaldehyde at $4^{\circ} \mathrm{C}$ for over $24 \mathrm{~h}$, dehydrated and embedded in paraffin following standard protocols by Servicebio, Inc. (Wuhan Servicebio Technology Co., Ltd., Wuhan, Hubei, China). The samples were stored and sectioned at room temperature; $4 \mu \mathrm{m}$ sections were incubated in a $65^{\circ} \mathrm{C}$ oven for $2 \mathrm{~h}$ and de-paraffinized by washing with xylene and a descending alcohol gradient $(100,100,85$ and $75 \%$ for immunohistochemistry staining; 100, 95, 90, 80 and $70 \%$ for Masson trichrome staining) prior to staining.

Immunohistochemistry and Masson trichrome staining were performed using mouse-anti-human monoclonal transforming growth factor (TGF)- $\beta 1$ antibodies $(1: 200$, cat. no. GB11179, Servicebio, Inc., Wuhan Servicebio Technology Co., Ltd.) and Masson trichrome (cat. no. PT003; 
Table IV. miRs with significantly different expression, as identified by miR sequencing.

A, Upregulated genes

\begin{tabular}{lcc}
\hline miR & $\begin{array}{c}\text { Fold change } \\
\text { (scar/normal) }\end{array}$ & P-value $^{\mathrm{a}}$ \\
\hline hsa-miR-129-5p & 3.75 & 0.026 \\
hsa-miR-9-5p & 2.08 & 0.003 \\
hsa-miR-374a-3p & 1.92 & 0.039 \\
hsa-miR-374b-3p & 1.42 & 0.008 \\
hsa-miR-128-3p & 1.19 & 0.014 \\
\hline
\end{tabular}

B, Downregulated genes

\begin{tabular}{lcc}
\hline & $\begin{array}{c}\text { Fold change } \\
\text { miR }\end{array}$ & \\
\hline hscar/normal) & P-value $^{\mathrm{a}}$ \\
hsa-miR-26a-5p & 0.91 & 0.004 \\
hsa-miR-223-3p & 0.90 & 0.019 \\
hsa-miR-3158-3p & 0.83 & 0.043 \\
hsa-miR-20a-5p & 0.78 & 0.025 \\
hsa-miR-92a-3p & 0.72 & 0.035 \\
hsa-miR-29c-3p & 0.70 & 0.039 \\
hsa-miR-194-5p & 0.69 & 0.002 \\
hsa-miR-125b-5p & 0.67 & 0.000 \\
hsa-miR-29b-3p & 0.67 & 0.005 \\
hsa-miR-192-5p & 0.64 & 0.008 \\
hsa-miR-10a-3p & 0.63 & 0.041 \\
hsa-miR-182-5p & 0.62 & 0.023 \\
hsa-miR-106a-5p & 0.51 & 0.016 \\
hsa-miR-183-5p & 0.46 & 0.038 \\
hsa-miR-6720-3p & 0.45 & 0.013 \\
hsa-miR-96-5p & 0.38 & 0.038 \\
hsa-miR-486-5p & 0.33 & 0.037 \\
hsa-miR-363-3p & 0.27 & 0.035 \\
hsa-miR-135a-5p & 0.26 & 0.014 \\
hsa-miR-4284 & 0.19 & 0.004 \\
\hline
\end{tabular}

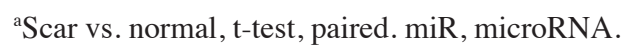

Shanghai Bogoo Biotechnology. Co., Ltd., Shanghai, China) following the manufacturer's protocols. Secondary antibody staining involved the DAKO REAL EnVision Detection System, Peroxidase/DAB, Rabbit/Mouse (cat. no. K5007; Agilent Technologies, Inc., Santa Clara, CA, USA) and a Masson staining kit (cat. no. G1006; Servicebio, Inc., Wuhan Servicebio Technology Co., Ltd.) according to the manufacturer's protocols.

\section{Results}

miRNA expression results based on sequencing data. All sequencing samples were evaluated for RNA quality (Table II)
Table V. Comparison between miR sequencing and qPCR sequencing.

\begin{tabular}{lcc}
\hline & $\begin{array}{c}\text { Average change } \\
\text { by miR sequencing } \\
\text { miR }\end{array}$ & $\begin{array}{c}\text { Average change } \\
\text { by qPCR } \\
\text { (scar/normal) }\end{array}$ \\
\hline has-miR-129-5p & 3.75 & 2.13 \\
hsa-miR-654-5p & 2.06 & 1.57 \\
hsa-miR-31-3p & 1.55 & 1.08 \\
hsa-miR-433-3p & 1.42 & 2.36 \\
hsa-miR-329-3p & 1.32 & 2.46 \\
hsa-let-7b-3p & 1.17 & 2.71 \\
hsa-miR-379-3p & 1.17 & 2.23 \\
hsa-miR-26a-5p & 0.91 & 1.13 \\
hsa-miR-744-5p & 0.84 & 2.52 \\
hsa-miR-223-3p & 0.83 & 0.54 \\
hsa-miR-20a-5p & 0.72 & 0.98 \\
hsa-miR-92a-3p & 0.70 & 1.12 \\
hsa-miR-29c-3p & 0.69 & 1.74 \\
hsa-miR-125b-5p & 0.67 & 1.70 \\
hsa-miR-29c-5p & 0.67 & 3.01 \\
hsa-miR-192-5p & 0.63 & 2.35 \\
hsa-miR-106a-5p & 0.46 & 1.19 \\
hsa-miR-183-5p & 0.45 & 2.22 \\
hsa-miR-96-5p & 0.33 & 1.77 \\
hsa-miR-486-5p & 0.27 & 1.84 \\
hsa-miR-4284 & 0.17 & 2.03 \\
\hline
\end{tabular}

miR, microRNA; qPCR, quantitative polymerase chain reaction.

and an miRNA expression profile was generated to identify differentially expressed miRNAs in scar tissues of patients with urethral stricture. Out of the 1,620 human miRNAs that were sequenced, $55 \mathrm{miRNAs}$ were upregulated (fold change $>2$ ) and 39 were downregulated (fold change $<0.5$ ).

A total of 26 differentially expressed miRNAs were identified, with 5 upregulated and 21 downregulated ( $\mathrm{P}<0.05$; Fig. 1). The fold changes and P-values were summarized in Table IV.

Valitation of miRNAs with qPCR. qPCR was performed for miRNAs with significant changes based on the miRNA sequencing data (Fig. 2). miRNA upregulation identified by miRNA sequencing was validated by qPCR $(\mathrm{P}=0.51$; paired t-test comparisons; $n=7$ ), while miRNA downregulation identified by miRNA sequencing was quite different from the data generated by qPCR $(\mathrm{P}<0.01$; paired t-test comparisons; $n=15$; Table V). Among these, hsa-miR-129-5p was overexpressed in scar tissue compared with normal tissue, although the difference was not significant. Hsa-miR-223-3p and hsa-miR-20a-5p expression was slightly decreased in scar tissues compared with normal tissues. Hsa-miR-26a-5p, hsa-miR-92a-3p, hsa-miR-29c-3p and hsa-miR-125b-5p demonstrated slight changes in expression, but showed opposite regulation patterns as validated by qPCR. Given the fact that sample size was small and the materials harvested were limited in the present study, it was not possible to generate 


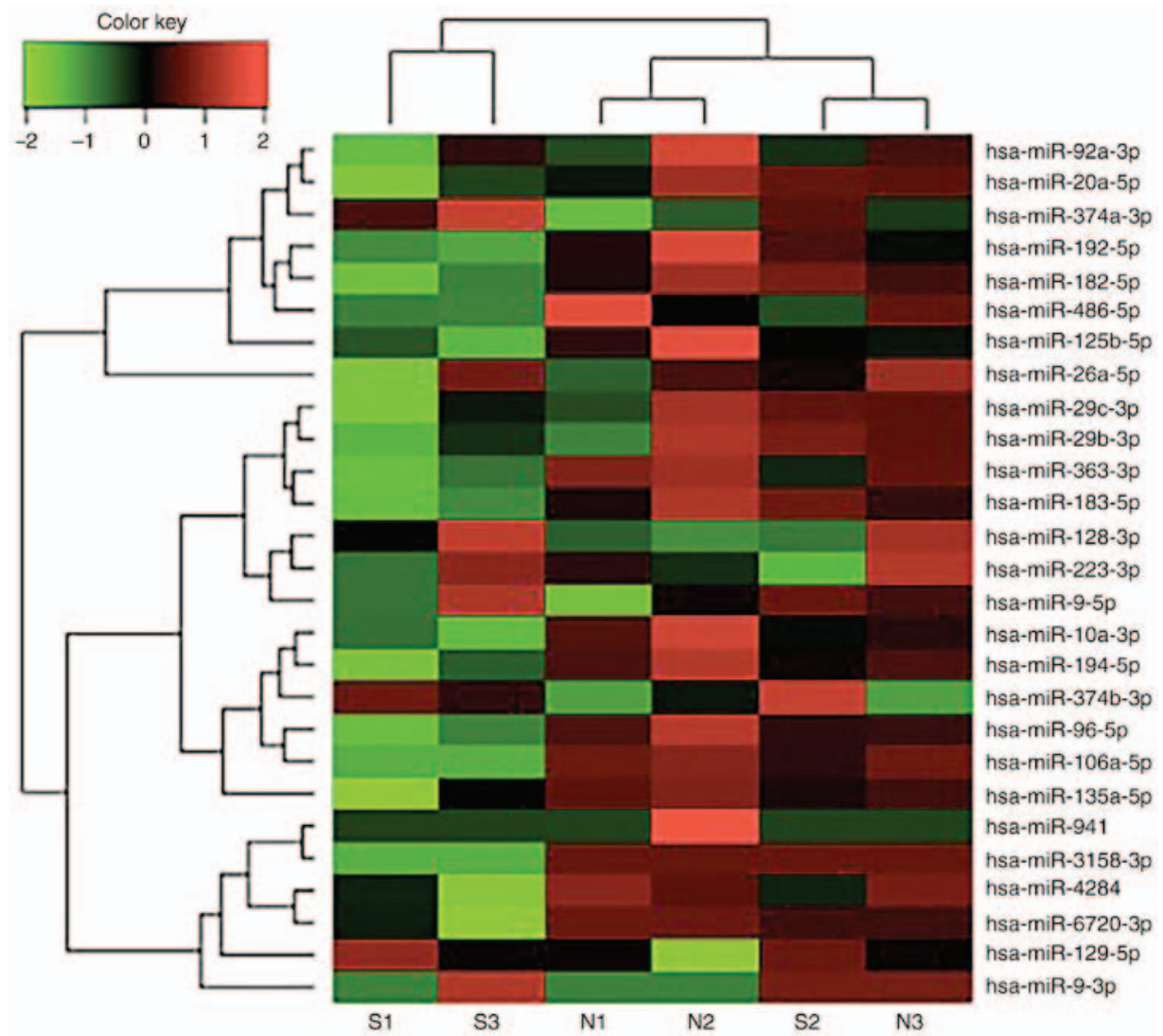

Figure 1. Hierarchical clustering of 26 miRs with significant changes in expression (fold change $>2$ or $<0.5$; $\mathrm{P}<0.05$ ) in the urethral scar and normal urethral tissue groups. Red indicates high expression, and green indicates low expression. miR, microRNA.

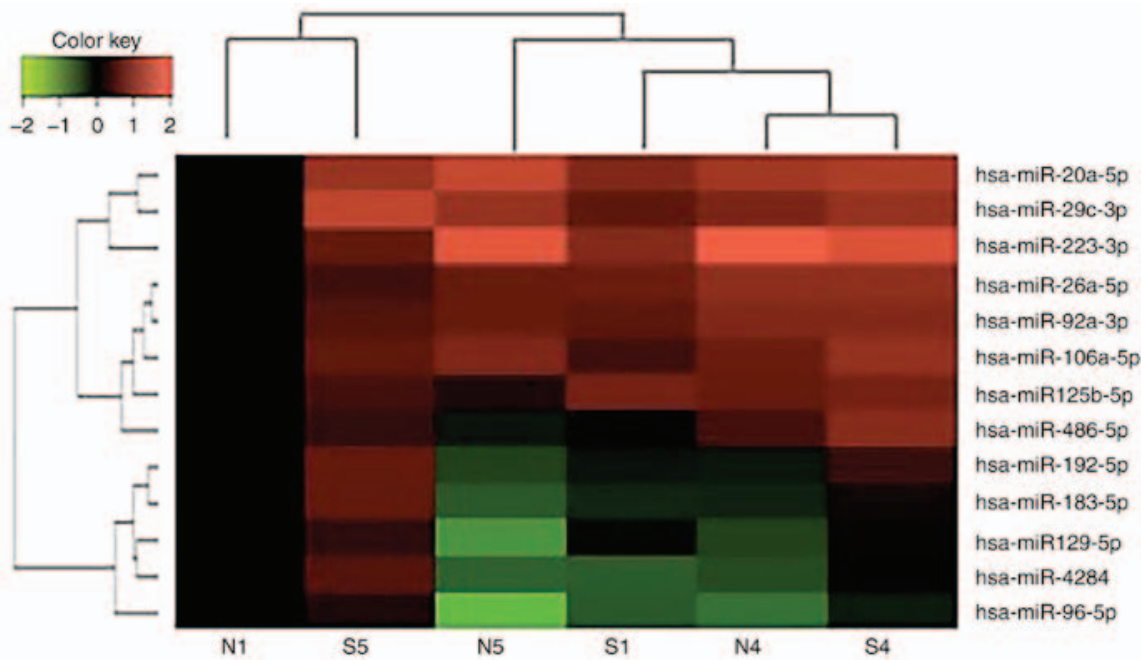

Figure 2. Validation of sequencing data by real time quantitative polymerase chain reaction. Triplicate assays were performed for each RNA sample. Red indicates high expression, and green indicates low expression. miR, microRNA.

certain conclusions or rule out the possibility of sample variety in this case. Further functional validation will be required to confirm the involvement of these candidate miRNAs in fibrosis, scar formation and PFUDD.

GO and KEGG pathway analysis. The predicted biological processes, molecular function, cellular components and KEGG pathways associated with miR-129-5p, miR-9-5p, miR-6720-3p, miR-363-3p and miR-135a-5p were analyzed (Figs. 3-7). As expected, these miRNAs were predicted to be most frequently involved in 'regulation of transcription' and 'RNA metabolic process'. miR-129-5p, miR-9-5p and miR-135a-5p were predicted to function in ion binding, while miR-6720-3p in both ion binding and DNA binding. miR-363-3p was the only miRNA predicted to molecularly regulate transcription and protein kinase activity. Cellular component analysis suggested that miR-129-5p, miR-9-5p and miR-135a-5p were associated with 'plasma membrane', miR-6720-3p in 'membrane-enclosed 

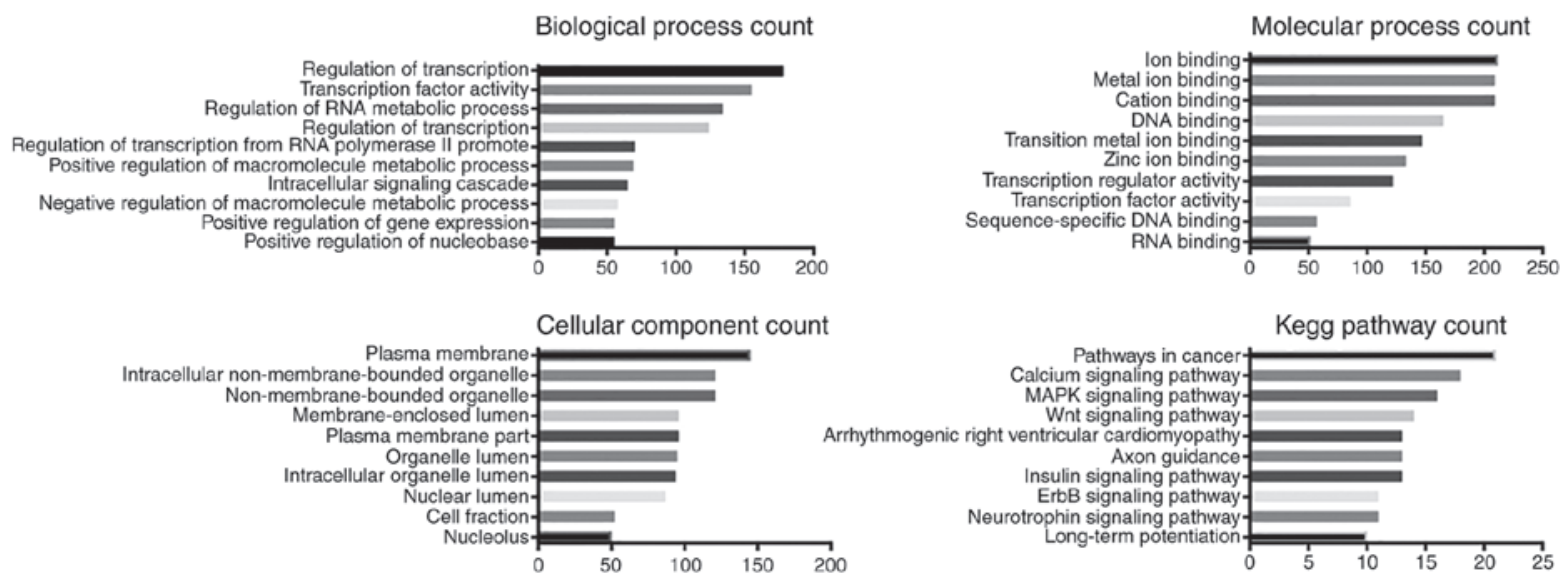

Figure 3. Biological process, molecular function, cellular component and Kyoto Encyclopedia of Genes and Genomes pathways of microRNA-129-5p. MAPK, mitogen-activated protein kinase.

Biological process count

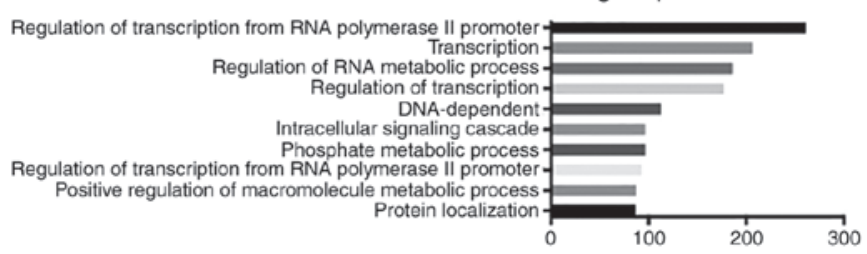

Cellular component count

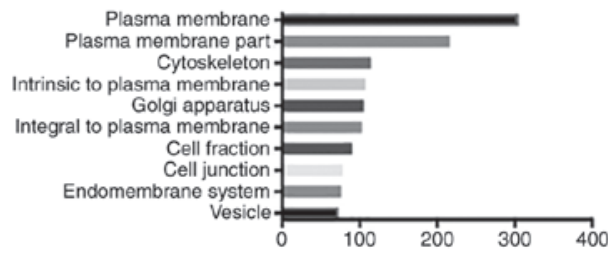

Molecular process count

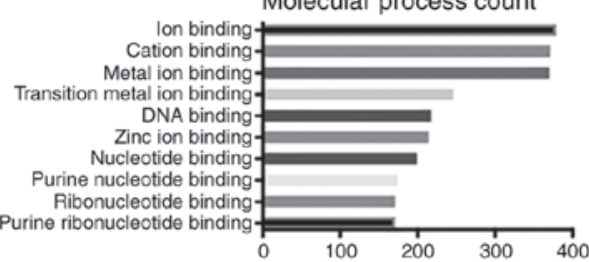

Kegg pathway count

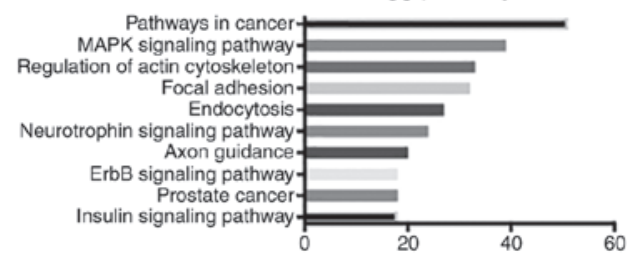

Figure 4. Biological process, molecular function, cellular component and Kyoto Encyclopedia of Genes and Genomes pathways of microRNA-9-5p. MAPK, mitogen-activated protein kinase.

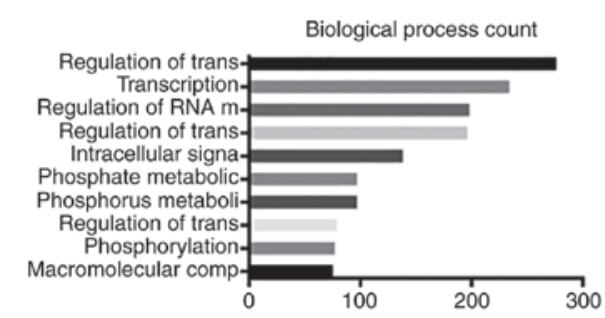

Cellular component count

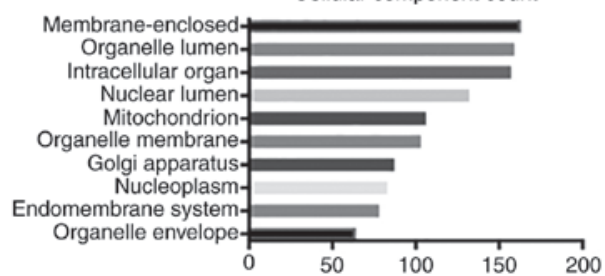

Molecular process count

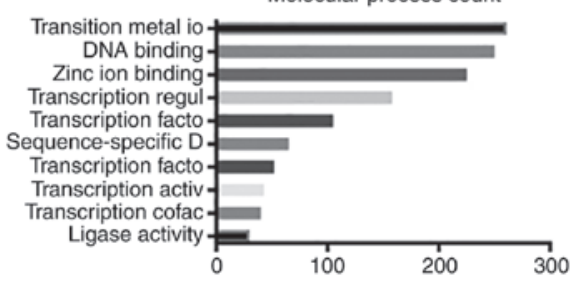

Kegg pathway count

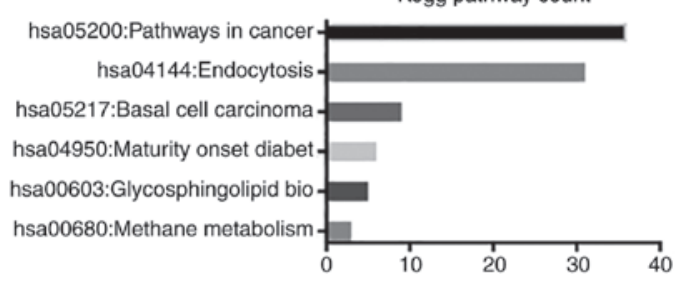

Figure 5. Biological process, molecular function, cellular component and Kyoto Encyclopedia of Genes and Genomes pathways of microRNA-6720-3p.

lumen', and miR-363-3p in 'non-membrane bounded organelles'. KEGG pathway analysis indicated that four miRNAs (miR-129-5p, miR-9-5p, miR-6720-3p and miR-135a-5p) may regulate pathways in cancer.
TGF- $\beta$ and collagen are upregulated in urethral scar tissue, but not normal tissue. TGF- $\beta$ signaling has been confirmed to be involved in fibrosis. To investigate whether TGF- $\beta$ signaling was affected in scar tissues, immunostaining for TGF- $\beta 1$ 

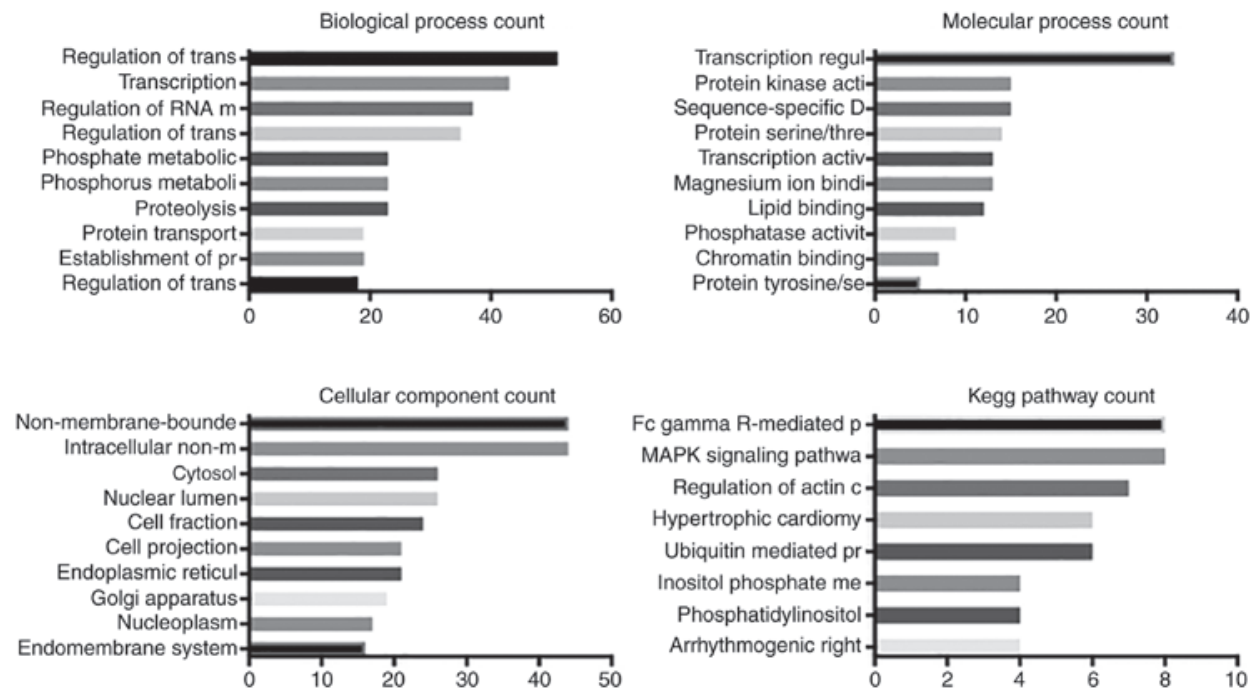

Figure 6. Biological process, molecular function, cellular component and Kyoto Encyclopedia of Genes and Genomes pathways of microRNA-363-3p.
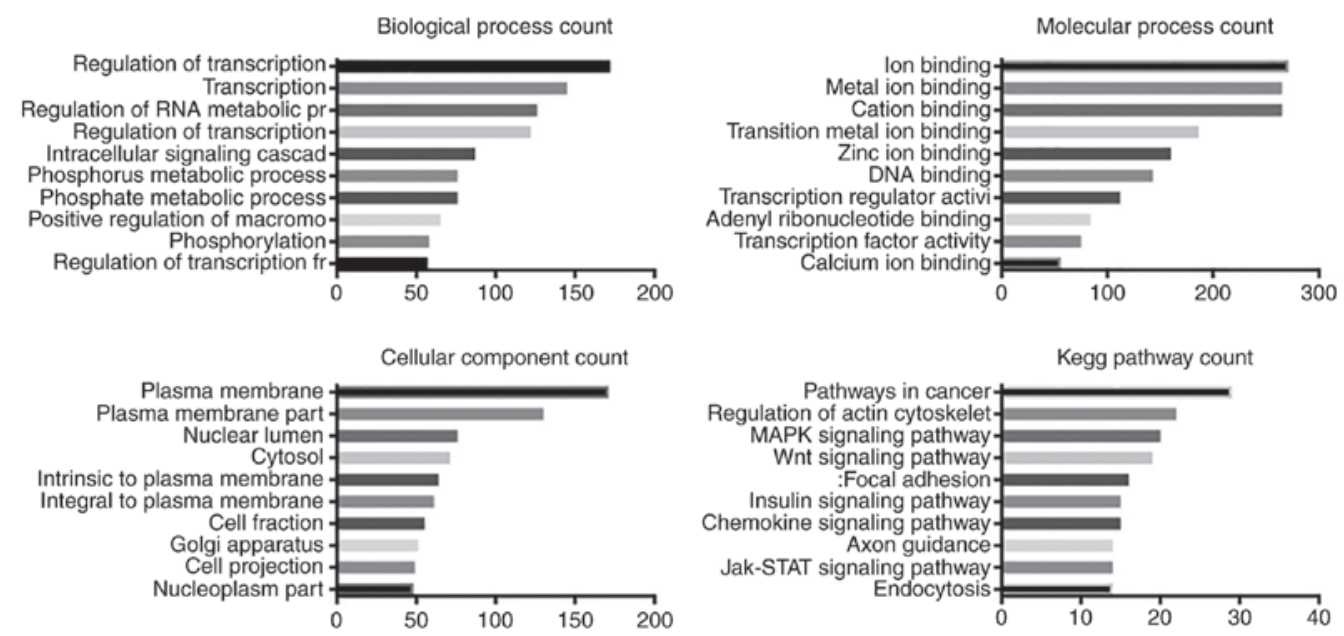

Figure 7. Biological process, molecular function, cellular component and Kyoto Encyclopedia of Genes and Genomes pathways of microRNA-135a-5p. MAPK, mitogen-activated protein kinase; Jak-STAT, Janus kinase-signal transducer and activator of transcription.

protein was performed. As expected, TGF- $\beta 1$ expression was increased in urethral scar tissues compared with normal tissues from the same patient (Fig. 8A and B). Similarly, dense collagen distribution and irregular fiber alignment was observed in urethral scar tissues, but not in normal tissue (Fig. 8C and D).

\section{Discussion}

miR-129-5p potentially regulates 17 genes that are directly associated with TGF- $\beta$ pathway members (Fig. 9), indicating that it may serve an important function in fibrosis through the regulation of TGF- $\beta$ signalling. miR-129-5p has previously been reported to be significantly downregulated following TGF- $\beta 1$ treatment in kidney proximal tubular cells (23). In addition, miR-129-5p is downregulated in fibroblasts in systemic sclerosis (or scleroderma, an acquired disorder that typically results in fibrosis of the skin and internal organs), and upregulated miR-129-5p (induced by interleukin-17A) suppresses $\alpha 1$ collagen protein expression levels in normal fibroblasts (24). The present study suggested that miR-129-5p is upregulated in scar tissues from patients with PFUDD compared with normal tissues, and further analysis is required to assess whether the downstream targets in TGF- $\beta$ pathway are functionally affected, and whether miR-129-5p regulation is driving the fibrosis or results from fibrosis as a feedback effect.

Besides its fibrosis-associated functions, miR-129-5p has also been reported as a regulator of epithelial-to-mesenchymal transition (EMT) in epithelial cells (23). miR-129-5p is significantly downregulated in various types of cancer, including lung cancer, breast cancer, gastric cancer and glioblastoma, and overexpression of miR-129-5R suppresses cell viability, proliferation, migration and invasion, while promoting cell apoptosis (25-28). miR-129-5p has also been demonstrated to negatively regulate a specific circular RNA (hsa-circ-0005986) and Notch1, and downregulated hsa-circ-0005986 may promote the proliferation of hepatocellular carcinoma cells (29). Investigation of miR-129-5p and its potential targets through TGF- $\beta$-independent regulations in PFUDD may prove interesting.

miR-9-5p expression is increased in lung tissues from patients with idiopathic pulmonary fibrosis and in the 

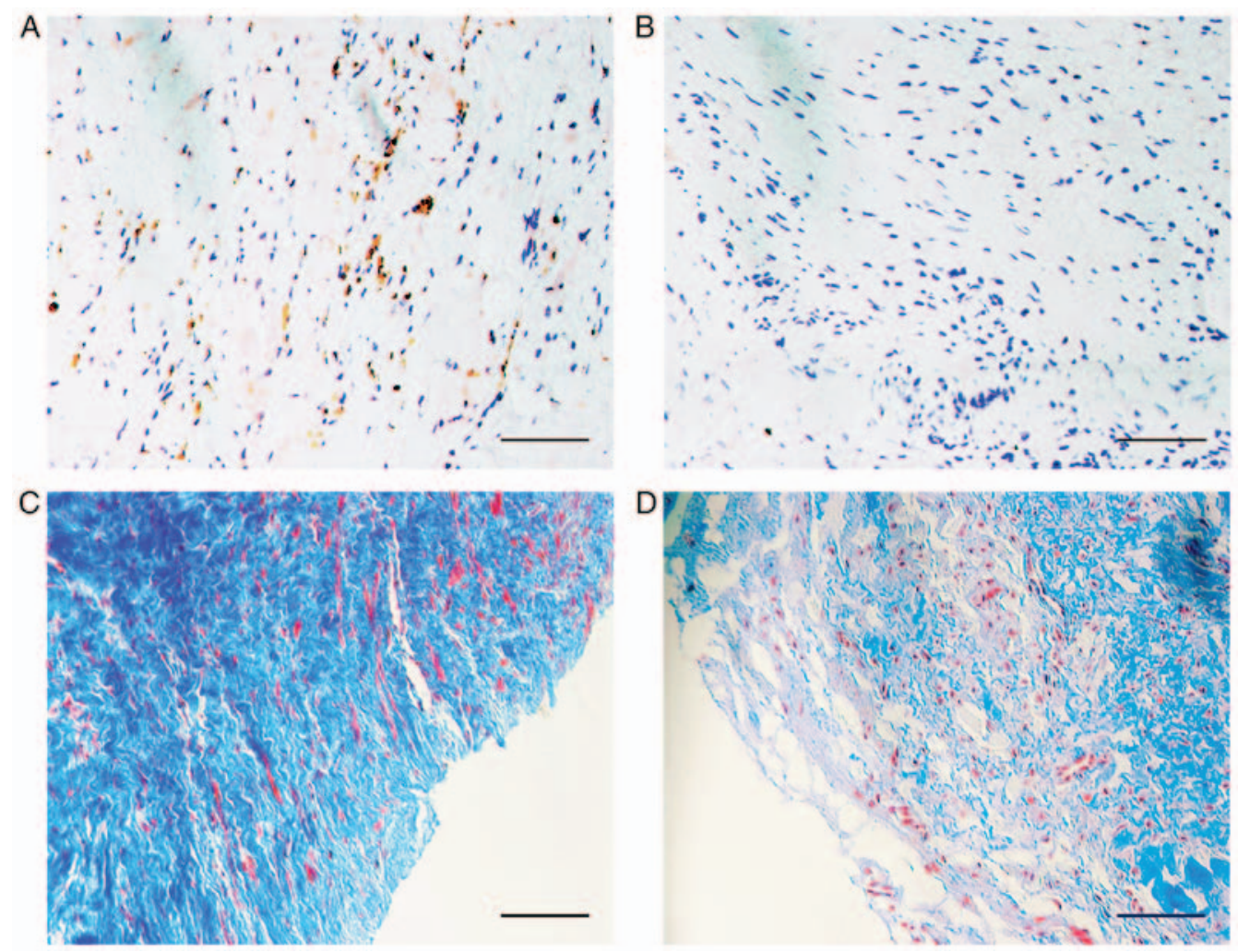

Figure 8. TGF- $\beta 1$ and collagen are overexpressed in urethral scar tissues compared with normal tissues. Anti-TGF- $\beta 1$ (brown) immunostating in (A) urethral scar tissue or (B) normal urethral tissue. Masson trichrome staining in (C) urethral scar tissue or in (D) normal urethral tissue. Collagen (blue), muscle fiber (red), cytoplasm (light red/pink) were labeled with their corresponding colors. Scale bar, $100 \mu \mathrm{m}$. TGF- $\beta 1$, transforming growth factor- $\beta 1$.

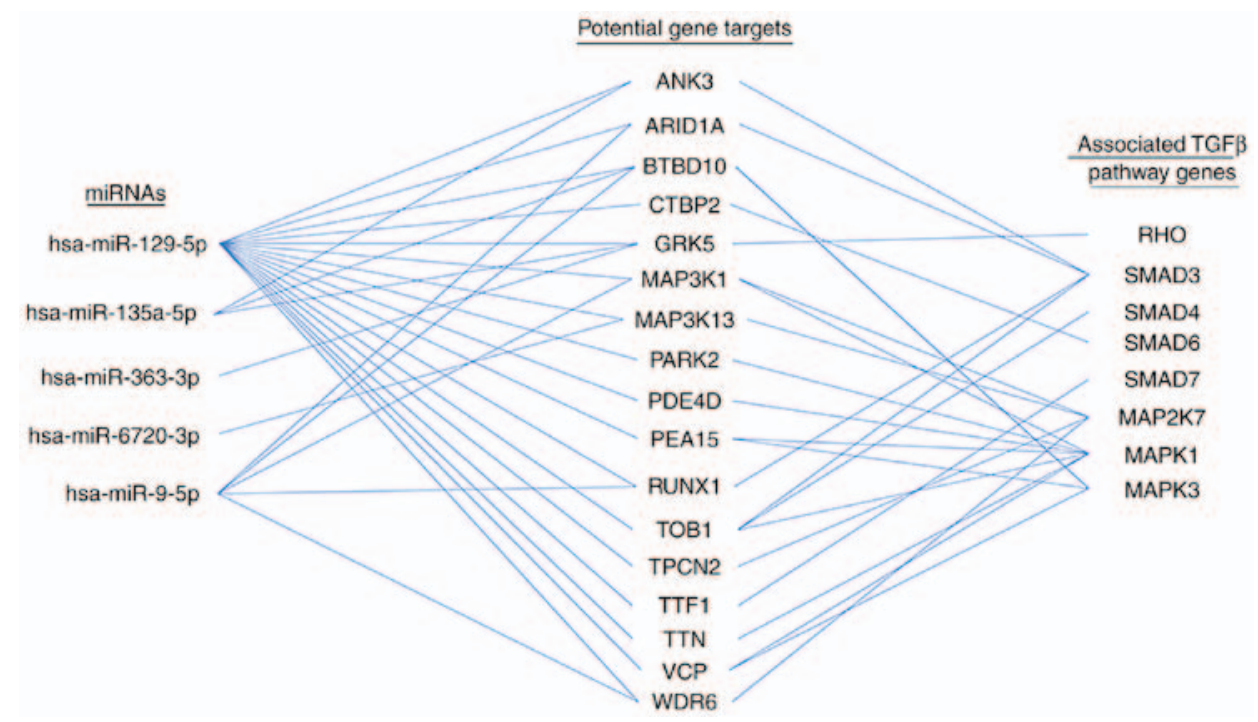

Figure 9. Crosstalk between miRNAs and target genes associated with TGF- $\beta 1$ regulated fibrosis. MiRNAs and genes that are 'hotspots' (interacting with multiple targets or predicted interacting partners) are in bold. miRNAs/miRs, microRNAs; TGF- $\beta 1$, transforming growth factor- $\beta 1$.

lungs of mice with bleomycin-induced lung fibrosis (30). Overexpression of miR-9-5p inhibits TGF- $\beta 1$-induced lung fibrosis, and inhibition of miR-9-5p amplifies the transformation of human lung fibroblasts into myofibroblasts (30). Overexpression of hsa-miR-9-5p may prevent fibrogenesis in skin fibrosis, mediated by suppressing TGF- $\beta$ signaling, and inhibition of miR-9-5p results in increased expression of fibrosis markers (31). Hsa-miR-9-5p is upregulated in TGF- $\beta 1$-treated human dermal fibroblasts (31). Overexpression of miR-9-5p significantly delays TGF- $\beta$-dependent transformation of dermal fibroblasts, decreases the expression of the ECM proteins collagen, type I, $\alpha 1$ and fibronectin, the amount of secreted collagen proteins and the expression of the archetypal myofibroblast marker $\alpha$-smooth muscle actin (31). In contrast to this, specific inhibition of miR-9-5p resulted in enhanced presence of fibrosis markers (31). The upregulation of hsa-miR-9-5p observed in the present study may have been an induced auto-suppression effect of fibrosis, and an analysis 
with larger cohort and further validation may be helpful to understand its function during the process.

Little is known about the involvement of miR-363-3p, miR-135a-5p or miR-6720-3p in fibrosis regulation. The majority of the research concerning these three miRNAs focuses on their function in carcinogenesis. For example, miR-363-3p is downregulated in tissues from patients with non-small cell lung cancer and gallbladder cancer, and in cluster of differentiation 133-positive cancer stem-like cells isolated from patients with larynx cancer (32-34). miR-363-3p significantly inhibits cell growth in vitro and in vivo in lung cancer cells, and induces cell cycle arrest and promote cell apoptosis, potentially through proliferating cell nuclear antigen and its ability to inhibit the activation of the mechanistic target of rapamycin and extracellular signal-regulated kinase (ERK) signaling pathways $(33,35)$. In patients with prostate cancer, miR-363-3p expression was decreased in serum and its level was negatively correlated with risk of recurrence and progression, suggesting that it may serve as a potential diagnostic and prognostic biomarker for prostate cancer (36). Similarly, in colorectal cancer, miR-363-3p was downregulated in the tissues of patients, and its expression was negatively associated with metastatic status (37). miR-363-3p inhibits colorectal cancer cell migration and invasion and inhibits EMT, potentially through targeting SRY-box 4 (37). Inhibition of miR-363-3p induces cell cycle arrest and suppresses apoptosis in gallbladder cancer cells, potentially by targeting and competing with the long non-coding RNA metastasis associated lung adenocarcinoma transcript 1 in the regulation of the MCL1, BCL2 family apoptosis regulator gene (34).

miR-135a-5p is upregulated in liver biopsy samples from patients with chronic hepatitis $\mathrm{C}$ or hepatitis $\mathrm{C}$ virus (HCV), but is not induced by anti-viral response to HCV infection (38). By targeting and inducing silencing of protein tyrosine phosphatase receptor $\delta$, a tumor suppressor, miR-135a-5p may potentially drive $\mathrm{HCV}$-associated hepatocarcinogenesis (38).

miR-6720 has been reported to be upregulated in hepatocellular carcinoma cells following treatment with alternariol, a mycotoxin (39). However, not much evidence is available concerning its functions in TGF- $\beta$ regulated signaling transduction.

As a shared potential target of hsa-miR-129-5p, hsa-miR-135a-5p and hsa-miR-363-3p, the G protein-coupled receptor kinase 5 (GRK5) gene encodes a member of the guanine nucleotide-binding protein-coupled receptor kinase subfamily of the Ser/Thr protein kinase family. GRK5 mRNA and protein expression is increased in the lungs of patients with cystic fibrosis compared with normal donors (40). GRK5 has been identified as a regulator of aldosterone-mineralocorticoid receptor/angiotensin II type 1 receptor-mediated cardiomyocyte hypertrophy (41). Molecular analysis has revealed that overexpression of N-terminal GRK5 inhibits apoptosis and fibrosis, which are major pathogenic phenotypes that accompany cardiac hypertrophy, through nuclear factor- $\kappa \mathrm{B}$ signaling (42). Knockout of GRK5 inhibits cardiac fibrosis induced by metoprolol, a $\beta_{1}$-adrenergic receptor-selective blocker, and this regulation is dependent on $\beta$-arrestins but independent of G-protein signaling (43).

TGF- $\beta 1$ and collagen were overexpressed in scar tissues in the present study (Fig. 8), indicating that the TGF- $\beta$ pathway was upregulated. TGF- $\beta 1$ is a key regulatory molecule in the pathogenesis of fibrosis, and is frequently upregulated during this process $(44,45)$. Inhibition of TGF- $\beta 1$ or its receptors, including TGF- $\beta$ receptor 1 , may reverse the unilateral ureteral occlusion-induced increase of fibrotic biomarkers, including TGF- $\beta 1$, collagens and fibronectin, at the mRNA level (44). The increase of TGF- $\beta 1$ and collagen may result in excessive fibrosis in scar tissue, and it is unclear how the regulatory miRNAs and genes that regulate TGF- $\beta$ signaling are involved in this process. Potential crosstalk between miRNAs and their target genes are listed, together with the associated signaling molecules involved in TGF- $\beta 1$ regulated fibrosis (Fig. 9).

It is worth noting that mitogen-activated protein kinase 1, proliferation and apoptosis adaptor protein 15 , transducer of ERBB2, 1 and valosin containing protein gene products are predicted to interact with one or more TGF- $\beta$ pathway proteins, as presented in Fig. 9. Among the associated TGF- $\beta$ pathway genes, SMAD family member 3 , mitogen-activated protein kinase 7 and ERK1/2 are the most frequently targeted candidates for potential interactions. Given the fact that crosstalk between SMADs and the ERK signaling pathway is essential in fibrosis (46), further functional analysis focusing on those 'hotspots' in larger cohorts may prove interesting.

In conclusion, the present study sequenced the urethral scar and normal tissue from patients with urethral stricture. miRNA sequencing indicated significantly altered expression of hsa-miR-129-5p, hsa-miR-135a-5p, hsa-miR-363-3p, hsa-miR-6720-3p and hsa-miR-9-5p. Bioinformatics analysis revealed that these miRNAs and their potential target genes are associated with fibrosis in other diseases. These data may provide miRNA targets for future precision treatments for urethral stricture.

\section{Acknowledgements}

Financial support was obtained from the National Natural Science Fund of China (grant nos. 81470917 and 81700590), the Science and Technology Commission of Shanghai (grant no. 17410742800), and the Shanghai JiaoTong University Biomedical Engineering Cross Research Foundation (grant no. YG2017QN15).

\section{Competing interests}

The authors declare that they have no competing interests.

\section{References}

1. Tritschler S, Roosen A, Füllhase C, Stief CG and Rübben H: Urethral stricture: Etiology, investigation and treatments. Dtsch Arztebl Int 110: 220-226, 2013.

2. Osman NI, Hillary C, Bullock AJ, MacNeil S and Chapple CR: Tissue engineered buccal mucosa for urethroplasty: Progress and future directions. Adv Drug Deliv Rev 82-83: 69-76, 2015.

3. Hampson LA, McAninch JW and Breyer BN: Male urethral strictures and their management. Nat Rev Urol 11: 43-50, 2014.

4. Andrich DE, Dunglison N, Greenwell TJ and Mundy AR: The long-term results of urethroplasty. J Urol 170: 90-92, 2003.

5. Lumen N, Hoebeke P and Oosterlinck W: Urethroplasty for urethral strictures: Quality assessment of an in-home algorithm. Int J Urol 17: 167-174, 2010.

6. Nerli RB, Neelagund SE, Guntaka A, Patil S, Hiremath SC Jali SM, Vernekar R and Hiremath MB: Staged buccal mucosa urethroplasty in reoperative hypospadias. Indian J Urol 27 : 196-199, 2011. 
7. Zhang K, Qi E, Zhang Y, Sa Y and Fu Q: Efficacy and safety of local steroids for urethra strictures: A systematic review and meta-analysis. J Endourol 28: 962-968, 2014.

8. Dubey D: The current role of direct vision internal urethrotomy and self-catheterization for anterior urethral strictures. Indian J Urol 27: 392-396, 2011.

9. O'Reilly S: MicroRNAs in fibrosis: Opportunities and challenges. Arthritis Res Ther 18: 11, 2016.

10. Griffiths-Jones S: The microRNA registry. Nucleic Acids Res 32: D109-D111, 2004.

11. Griffiths-Jones S, Grocock RJ, van Dongen S, Bateman A and Enright AJ: miRBase: microRNA sequences, targets and gene nomenclature. Nucleic Acids Res 34: D140-D144, 2006.

12. Griffiths-Jones S, Saini HK, van Dongen S and Enright AJ: miRBase: Tools for microRNA genomics. Nucleic Acids Res 36: D154-D158, 2008.

13. Kozomara A and Griffiths-Jones S: miRBase: Annotating high confidence microRNAs using deep sequencing data. Nucleic Acids Res 42: D68-D73, 2014.

14. Kozomara A and Griffiths-Jones S: miRBase: Integrating microRNA annotation and deep-sequencing data. Nucleic Acids Res 39: D152-D157, 2011.

15. Livak KJ and Schmittgen TD: Analysis of relative gene expression data using real-time quantitative PCR and the $2^{-\Delta \Delta C}$ method. Methods 25: 402-408, 2001.

16. Lewis BP, Burge CB and Bartel DP: Conserved seed pairing, often flanked by adenosines, indicates that thousands of human genes are microRNA targets. Cell 120: 15-20, 2005.

17. Enright AJ, John B, Gaul U, Tuschl T, Sander C and Marks DS MicroRNA targets in Drosophila. Genome Biol 5: R1, 2003.

18. Huang da W, Sherman BT and Lempicki RA: Systematic and integrative analysis of large gene lists using DAVID bioinformatics resources. Nat Protoc 4: 44-57, 2009.

19. Huang da W, Sherman BT and Lempicki RA: Bioinformatics enrichment tools: Paths toward the comprehensive functional analysis of large gene lists. Nucleic Acids Res 37: 1-13, 2009.

20. Ashburner M, Ball CA, Blake JA, Botstein D, Butler H, Cherry JM, Davis AP, Dolinski K, Dwight SS, Eppig JT, et al: Gene ontology: Tool for the unification of biology. The Gene Ontology Consortium. Nat Genet 25: 25-29, 2000.

21. The Gene Ontology Consortium: Expansion of the Gene Ontology knowledgebase and resources. Nucleic Acids Res 45: D331-D338, 2017.

22. Kanehisa M and Goto S: KEGG: Kyoto encyclopedia of genes and genomes. Nucleic Acids Res 28: 27-30, 2000.

23. Li Y, An H, Pang J, Huang L, Li J and Liu L: MicroRNA profiling identifies miR-129-5p as a regulator of EMT in tubular epithelial cells. Int J Clin Exp Med 8: 20610-20616, 2015.

24. Nakashima T, Jinnin M, Yamane K, Honda N, Kajihara I, Makino T, Masuguchi S, Fukushima S, Okamoto Y, Hasegawa M, et al: Impaired IL-17 signaling pathway contributes to the increased collagen expression in scleroderma fibroblasts. J Immunol 188: 3573-3583, 2012.

25. Zhang Y, An J, Lv W, Lou T, Liu Y and Kang W: miRNA-129-5p suppresses cell proliferation and invasion in lung cancer by targeting microspherule protein 1, E-cadherin and vimentin. Oncol Lett 12: 5163-5169, 2016.

26. $\mathrm{Xu} \mathrm{H}, \mathrm{Hu}$ Y and Qiu W: Potential mechanisms of microRNA-129-5p in inhibiting cell processes including viability, proliferation, migration and invasiveness of glioblastoma cells U87 through targeting FNDC $3 B$. Biomed Pharmacother 87: 405-411, 2017.

27. Luan QX, Zhang BG, Li XJ and Guo MY: MiR-129-5p is downregulated in breast cancer cells partly due to promoter $\mathrm{H} 3 \mathrm{~K} 27 \mathrm{~m} 3$ modification and regulates epithelial-mesenchymal transition and multi-drug resistance. Eur Rev Med Pharmacol Sci 20: 4257-4265, 2016 .

28. Jiang Z, Wang H, Li Y, Hou Z, Ma N, Chen W, Zong Z and Chen S: MiR-129-5p is down-regulated and involved in migration and invasion of gastric cancer cells by targeting interleukin- 8 Neoplasma 63: 673-680, 2016.

29. Fu L, Chen Q, Yao T, Li T, Ying S, Hu Y and Guo J: Hsa circ 0005986 inhibits carcinogenesis by acting as a miR-129-5p sponge and is used as a novel biomarker for hepatocellular carcinoma. Oncotarget 8: 43878-43888, 2017.

30. Fierro-Fernández M, Busnadiego Ó, Sandoval P, Espinosa-Díez C, Blanco-RuizE, Rodríguez M,Pian H, Ramos R,López-Cabrera M, García-Bermejo ML and Lamas S: miR-9-5p suppresses pro-fibrogenic transformation of fibroblasts and prevents organ fibrosis by targeting NOX4 and TGFBR2. EMBO Rep 16: 1358-1377, 2015.
31. Miguel V, Busnadiego O, Fierro-Fernández M and Lamas S: Protective role for miR-9-5p in the fibrogenic transformation of human dermal fibroblasts. Fibrogenesis Tissue Repair 9: 7, 2016.

32. Karatas OF, Suer I, Yuceturk B, Yilmaz M, Oz B, Guven G, Cansiz H, Creighton CJ, Ittmann $M$ and Ozen M: Identification of microRNA profile specific to cancer stem-like cells directly isolated from human larynx cancer specimens. BMC Cancer 16: 853,2016

33. Wang Y, Chen J, Lin Z, Cao J, Huang H, Jiang Y, He H, Yang L, Ren N and Liu G: Role of deregulated microRNAs in non-small cell lung cancer progression using fresh-frozen and formalin-fixed, paraffin-embedded samples. Oncol Lett 11: 801-808, 2016.

34. Wang SH, Zhang WJ, Wu XC, Weng MZ, Zhang MD, Cai Q, Zhou D, Wang JD and Quan ZW: The lncRNA MALAT1 functions as a competing endogenous RNA to regulate MCL-1 expression by sponging miR-363-3p in gallbladder cancer. J Cell Mol Med 20: 2299-2308, 2016.

35. Wang Y, Chen T, Huang H, Jiang Y, Yang L, Lin Z, He H, Liu T, Wu B, Chen J, et al: miR-363-3p inhibits tumor growth by targeting PCNA in lung adenocarcinoma. Oncotarget 8 : 20133-20144, 2017.

36. Cochetti G, Poli G, Guelfi G, Boni A, Egidi MG and Mearini E: Different levels of serum microRNAs in prostate cancer and benign prostatic hyperplasia: Evaluation of potential diagnostic and prognostic role. OncoTargets Ther 9: 7545-7553, 2016.

37. Hu F, Min J, Cao X, Liu L, Ge Z, Hu J and Li X: MiR-363-3p inhibits the epithelial-to-mesenchymal transition and suppresses metastasis in colorectal cancer by targeting Sox4. Biochem Biophys Res Commun 474: 35-42, 2016.

38. Van Renne N, Roca Suarez AA, Duong FH, Gondeau C, Calabrese D, Fontaine N, Ababsa A, Bandiera S, Croonenborghs T, Pochet N, et al: miR-135a-5p-mediated downregulation of protein tyrosine phosphatase receptor delta is a candidate driver of HCV-associated hepatocarcinogenesis. Gut: gutjnl-2016-312270, 2017.

39. Vejdovszky K, Sack M, Jarolim K, Aichinger G, Somoza MM and Marko D: In vitro combinatory effects of the Alternaria mycotoxins alternariol and altertoxin II and potentially involved miRNAs. Toxicol Lett 267: 45-52, 2017.

40. Mak JC, Chuang TT, Harris CA and Barnes PJ: Increased expression of $\mathrm{G}$ protein-coupled receptor kinases in cystic fibrosis lung. Eur J Pharmacol 436: 165-172, 2002.

41. Cannavo A, Liccardo D, Eguchi A, Elliott KJ, Traynham CJ, Ibetti J, Eguchi S, Leosco D, Ferrara N, Rengo G and Koch WJ: Myocardial pathology induced by aldosterone is dependent on non-canonical activities of $\mathrm{G}$ protein-coupled receptor kinases. Nat Commun 7: 10877, 2016.

42. Sorriento D, Santulli G, Fusco A, Anastasio A, Trimarco B and Iaccarino G: Intracardiac injection of AdGRK5-NT reduces left ventricular hypertrophy by inhibiting NF- $\mathrm{B}$-dependent hypertrophic gene expression. Hypertension 56: 696-704, 2010.

43. Nakaya M, Chikura S, Watari K, Mizuno N, Mochinaga K, Mangmool S, Koyanagi S, Ohdo S, Sato Y, Ide T, et al: Induction of cardiac fibrosis by $\beta$-blocker in $\mathrm{G}$ protein-independent and $\mathrm{G}$ protein-coupled receptor kinase 5/ $\beta$-Arrestin2-dependent signaling pathways. J Biol Chem 287: 35669-35677, 2012.

44. Richards TL, Minor K and Plato CF: Effects of transforming growth factor-beta (TGF- $\beta$ ) receptor I inhibition on renal biomarkers and fibrosis in unilateral ureteral occluded (UUO) Mice. FASEB J 31: 1030.1033, 2017.

45. Penn JW, Grobbelaar AO and Rolfe KJ: The role of the TGF- $\beta$ family in wound healing, burns and scarring: A review. Int $\mathrm{J}$ Burns Trauma 2: 18-28, 2012.

46. Zhan $M$ and Kanwar YS: Hierarchy of molecules in TGF- $\beta$ signaling relevant to myofibroblast activation and renal fibrosis. Am J Physiol Renal Physiol 307: F385-F387, 2014.

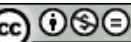

This work is licensed under a Creative Commons Attribution-NonCommercial-NoDerivatives 4.0 International (CC BY-NC-ND 4.0) License. 